\title{
Genetic and Environmental Dissections of Sub-Phenotypes of Metabolic Syndrome in the Chinese Population: A Twin-Based Heritability Study
}

\author{
Haiping Duan ${ }^{a, b *}$ Zengchang Pang ${ }^{a, b}$ Dongfeng Zhang ${ }^{b}$ Shuxia Li $^{c} \quad$ Torben A. Kruse $^{d}$ \\ Kirsten Ohm Kyvik ${ }^{c, e}$ Kaare Christensen ${ }^{c, d, f}$ Qihua Tan ${ }^{c, d^{*}}$ \\ ${ }^{a}$ Qingdao Center for Disease Control and Prevention, \\ ${ }^{\mathrm{b}}$ Department of Public Health, Qingdao University Medical College, Qingdao China \\ ${ }^{\mathrm{c}}$ Epidemiology, Institute of Public Health, University of Southern Denmark, \\ ${ }^{\mathrm{d}}$ Department of Clinical Genetics, Odense University Hospital, \\ ${ }^{\mathrm{e}}$ Institute for Regional Health Services Research, University of Southern Denmark, \\ ${ }^{f}$ Department of Clinical Biochemistry and Pharmacology, Odense University Hospital, Odense, Denmark
}

\section{Keywords}

Chinese twins - Metabolic syndrome - Heritability .

Univariate model · Bivariate model

\section{Summary}

Objective: We perform a comprehensive heritability study on multiple phenotypes related to metabolic syndrome using Chinese twins to assess the genetic and environmental effects in determining the variation and covariation of the phenotypes in the Chinese population. Methods: The studied sample contains 654 twins collected in the Qingdao municipality. A total of 10 phenotypes covering anthropometric measurements, plasma glucose levels, lipids, blood pressures etc. were examined. Univariate and bivariate structural equation models were fitted for assessing the genetic and environmental contributions. Results: The AE model combining additive genetic (A) and unique environmental (E) factors produced the best fit for all phenotypes except for triglyceride. Modest to high heritability estimates were obtained in univariate analysis ranging from 0.5 for total cholesterol to 0.78 for weight. The bivariate model estimated high genetic correlations between systolic and diastolic blood pressures, between total cholesterol and low density lipoprotein cholesterol, modest genetic correlations between BMI and blood pressures. No significant common environmental correlation was found between any

*These authors contributed equally to this work. pair of the phenotypes. Conclusions: Our results showed significant genetic contributions to the sub-phenotypes of metabolic syndrome. Although pleiotropic genetic control may exist for some physiologically similar phenotypes, our results do not support a common genetic mechanism among the phenotypes covered in our study.

\section{Introduction}

As one of the world's fastest developing economies, China is experiencing dramatic transformations in many social and economic conditions that are to alter the disease pattern in its population [1] due to changing lifestyle and dietary structure which are undergoing steady westernization. Although prevalence of overweight and obesity in China is relatively low $(15 \%)$ as compared with countries such as the USA, the country is experiencing an alarmingly rapid increase in the prevalence of obesity and related problems in the past decade. Without a proper intervention, the epidemic of metabolic disorders is to become a crucial public health issue in China [2].

As the sub-phenotypes of metabolic syndrome are more proximate to direct genetic control than the composite phenotype itself, genetic studies focusing on the sub-phenotypes should exhibit more power in helping understanding the etiology and development of the disorder. A number of studies have been published using twin and family data on multiple

\section{KARGER}

Fax +497614520714

Information@Karger.de

www.karger.com (c) 2011 S. Karger GmbH, Freiburg

$1662-4025 / 11 / 0042-0099 \$ 38.00 / 0$

Accessible online at:

www.karger.com/ofa 
metabolic phenotypes (glucose tolerance, BMI, waist-to-hip ratio (WHR), fasting plasma glucose (FPG), plasma glucose 120 min post oral glucose tolerance test ( $2 \mathrm{hPG}$ ), systolic blood pressure (SBP), diastolic blood pressure (DBP), high-density lipoprotein cholesterol (HDL-C), triglyceride (TG), low-density lipoprotein cholesterol (LDL-C) and total cholesterol (TC) etc.) in developed countries and mainly in Caucasians. Findings from these studies uniformly point to the genetic contributions in these phenotypes [3-11]. Although there have been sporadic studies in Chinese subjects on limited phenotypes $[12,13]$, it is unfortunate that a comprehensive heritability study with a broad coverage of phenotypes related to metabolic syndrome has been missing for the world largest Chinese population. It is well known that ethnic disparities in the pathophysiology and pathogenesis of metabolic diseases [14] can have an important impact in prevention and management [15] given the complex nature in disease development which involves both genetic and environmental factors (e.g. different food structure and lifestyles, lower baseline BMI in Chinese). Actually, previous studies have shown high prevalence of diabetes in Chinese living in Hong Kong [16, 17], Singapore [18] and Taiwan [19]. This article reports the results from our Chinese twin-based heritability study on multiple phenotypes associated with metabolic syndrome, including body measurements, blood pressure, glucose and lipid phenotypes, using both univariate and bivariate twin models.

\section{Material and Methods}

\section{Subjects}

Our twin sampling was based on the Qingdao Twin Registry [20] established in 2001 at the Qingdao Center for Disease Control and Prevention (CDC). Twins have been identified through a local disease control network, neighborhood/village committees and residence registry. Twins were invited to a clinical investigation if both co-twins were alive at the time of the study. Those who were pregnant, breastfeeding, had known diabetes and/or cardiovascular disease or were taking weight-reducing medicaments within 1 month were excluded after telephone interviews. A twin pair was also dropped if one of the co-twins declined participation. After informed consent, a total of 327 pairs of twins over 18 years of age (mean age 40 years) were collected in 5 districts in the Qingdao municipality (106 pairs of male like-sex twins, 157 pairs of female like-sex twins, 64 pairs of opposite-sex twins). The study was approved by the regional ethics committee and conducted according to the principles of the Helsinki Declaration.

\section{Methods}

Zygosity of all like-sex twin pairs was determined by 16 multiple short tandem repeats [21] at the central laboratory in the Qingdao Bloodbank.

All surveys including questionnaire, blood sample collection and anthropometric and laboratory measurements were done in Qingdao CDC. Weight and height were measured with the subject in lightweight clothes and their shoes removed. Weight was measured using a standing beam scale to the nearest $0.1 \mathrm{~kg}$, and height was measured using a vertical scale with a horizontal moving headboard to the nearest $0.1 \mathrm{~cm}$. BMI was calculated as weight $(\mathrm{kg})$ divided by square of height $(\mathrm{m})$. Circumferences were measured in the standing position. Waist circumference $(\mathrm{cm})$ was measured using a soft tape midway between the lowest rib and iliac crest to the nearest centimeter. Hip circumference was measured over the widest part of the gluteal region to the nearest centimeter. BP was taken by a standard procedure using mercurial table stand model sphygmomanometer. SBP was defined as Korotkof phase I (appearance of sound), and DBP was defined as Korotkof phase V (disappearance of sound). Three measurements were taken for each subject, with at least 1 min between each measurement. The mean of these three measurements was used in subsequent analyses. [5] Co-twins were examined on the same day after a 10-12 h overnight fast. Serum and plasma were separated from blood cells in the field within $30 \mathrm{~min}$ and kept frozen at $-80^{\circ} \mathrm{C}$. TC, TG, HDL-C and LDL-C phenotypes were measured on the semi-automatic analyzer. A WHO standard OGTT was carried out in the subjects who participated in 2008 (135 pairs, $65 \mathrm{MZ}$ pairs, $70 \mathrm{DZ}$ pairs). Peripheral blood was taken before oral glucose ingestion and $120 \mathrm{~min}$ later. If one or both twins in a pair had a FPG concentration over $6.7 \mathrm{mmol} / \mathrm{l}$ or a 120 -min blood glucose concentration higher than $11.1 \mathrm{mmol} / \mathrm{l}$, the twin pair was excluded to avoid residual confounding by undiagnosed diseases. Plasma glucose concentrations were analyzed by the glucose dehydrogenase oxidation method.

\section{Descriptive Analysis}

Data was entered and cleaned by Epidata 3.0 software (www.epidata.dk), analyzed using SPSS version 13.0 for Windows (SPSS, Chicago, IL, USA) for descriptive statistics and for calculating intraclass correlation coefficients (ICC) after adjusting for age and sex. All continuous phenotypes except SBP and DBP were log transformed to ensure approximation to normal distribution. Significance test on statistical differences between (monozygotic) MZ and dizygotic (DZ) twin correlation was performed first by transforming a correlation coefficient (r) using

$\mathrm{r}^{\prime}=(0.5) \log _{\mathrm{e}}\left|\frac{1+\mathrm{r}}{1-\mathrm{r}}\right|$

and then computing the test statistic as

$\mathrm{z}=\frac{\mathrm{r}_{\mathrm{mz}}^{\prime}-\mathrm{r}_{\mathrm{dz}}^{\prime}}{\sqrt{\frac{1}{\mathrm{n}_{\mathrm{mz}}-3}+\frac{1}{\mathrm{n}_{\mathrm{dz}}-3}}}$

where $r_{\mathrm{mz}}^{\prime}$ and $\mathrm{r}_{\mathrm{dz}}^{\prime}$ are the transformed coefficients, and $\mathrm{n}_{\mathrm{mz}}$ and $\mathrm{n}_{\mathrm{dz}}$, the number of pairs for MZ and DZ twins. $P$ values were obtained by referring the test statistic $\mathrm{z}$ to a standard normal distribution with one degree of freedom.

$r_{\mathrm{G}}$

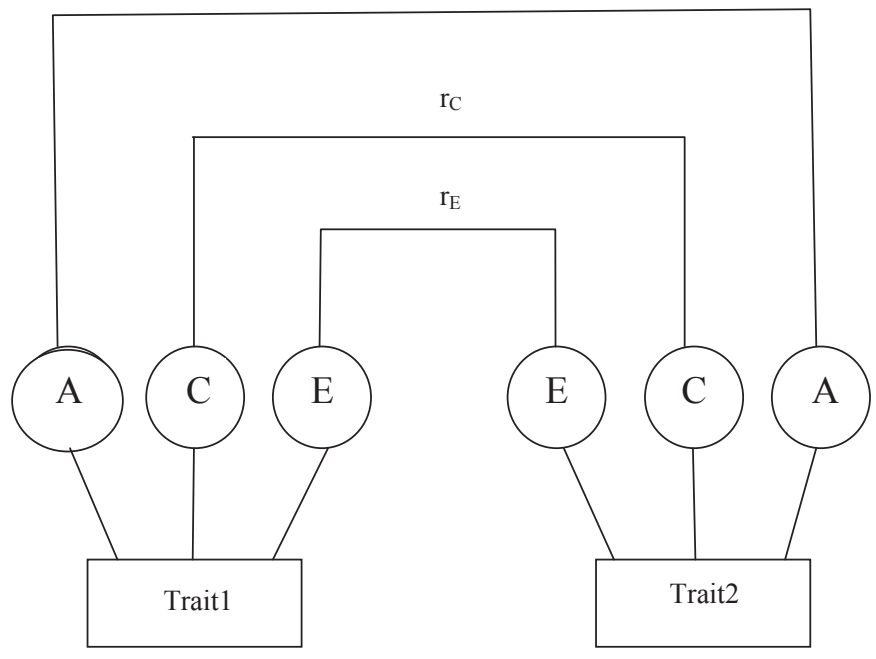

Fig. 1. Diagram showing the bivariate genetic model for trait 1 and trait2. $r_{G}, r_{C}$ and $r_{E}$ stand for genetic, common, and unique environmental correlations; A, C and $\mathrm{E}$ are variances due to genetic, common environmental, and unique environmental effects. 
Model-fitting and comparison were performed using the free software package Mx [22] (www.vcu.edu/mx). Since our DZ correlation is more than one half the MZ correlation for all phenotypes, an ACE model was preferred with $\mathrm{A}$ representing the additive genetic effects, $\mathrm{C}$ the shared and $\mathrm{E}$ the non-shared environmental effects. Comparisons on performances between the full ACE model and its nested models (AE, CE, E) were done using the likelihood ratio test. The likelihood ratio test calculates twice the difference in the log likelihoods for the full and the nested models which can be approximated by a chi-squared distribution with degree of freedom equaling the difference in the number of parameters in the two models. When no statistical significance is observed between two models, the parsimonious one is preferred. Comparison between $\mathrm{AE}$ and $\mathrm{CE}$ models was made using the Akaike Information Criterion (AIC). All models were fitted by adjusting for age and sex.

\section{Bivariate Genetic Models}

We use the bivariate Cholesky decomposition [23] model to assess the common genetic and environmental effects on phenotypic correlation among the intermediate phenotypes (fig. 1). The bivariate ACE model partitions the variation in liability for each of the variables into additive genetic, shared environmental and non-shared environmental components. Likewise, the covariance between two variables is also partitioned into that due to additive genetic, shared environmental and non-shared environmental parts. We report parameter estimates for all combinations of phenotype pairs that can be modeled together with their corresponding $95 \%$ confidence intervals ( $95 \% \mathrm{CI}$ ).

Table 1. Descriptive statistics for the twin samples

\begin{tabular}{llcc}
\hline Phenotype & $\begin{array}{l}\text { Number of twin } \\
\text { pairs }\end{array}$ & Median & 95\% range \\
\hline Age, years & 327 & 40.38 & $24.12-56.26$ \\
BMI, kg/m ${ }^{2}$ & 327 & 23.57 & $19.32-30.70$ \\
Weight, kg & 327 & 63 & $49.2-85$ \\
WHR & 272 & 0.83 & $0.73-0.96$ \\
FPG, mmol/l & 325 & 5.21 & $4.2-6.6$ \\
2hPG, mmol/l & 135 & 6.1 & $3.8-12$ \\
TC, mmol/l & 325 & 4.53 & $2.86-6.39$ \\
TG, mmol/l & 325 & 0.85 & $0.37-2.63$ \\
HDL-C, mmol/1 & 325 & 1.41 & $0.89-2.09$ \\
LDL-C, mmol/l & 325 & 2.6 & $1.36-3.91$ \\
SBP, mm Hg & 325 & 120 & $100-150$ \\
DBP, mm Hg & 325 & 80 & $62-100$ \\
\hline
\end{tabular}

Table 2. Intra-pair correlations after adjustment for age and sex

\begin{tabular}{lccc}
\hline Phenotype & MZ & DZ & p value \\
\hline BMI & 0.77 & 0.46 & $<0.001$ \\
Weight & 0.79 & 0.34 & $<0.001$ \\
WHR & 0.63 & 0.44 & $<0.05$ \\
FPG & 0.73 & 0.45 & $<0.001$ \\
2hPG & 0.65 & 0.36 & $<0.05$ \\
TC & 0.54 & 0.30 & $<0.05$ \\
TG & 0.56 & 0.46 & 0.206 \\
HDL & 0.56 & 0.38 & $<0.05$ \\
LDL & 0.64 & 0.40 & $<0.05$ \\
SBP & 0.60 & 0.46 & $<0.05$ \\
DBP & 0.64 & 0.35 & $<0.001$ \\
\hline
\end{tabular}

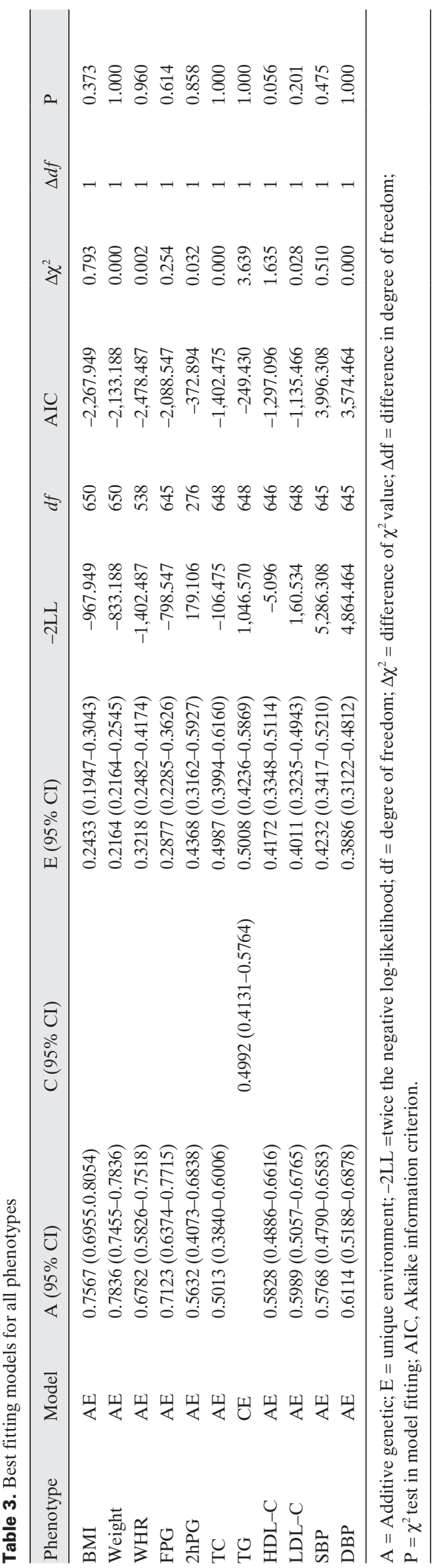


Table 4. he estimated genetic and environmental correlation coefficients for phenotype pairs

\begin{tabular}{llll}
\hline Phenotype & $\mathrm{r}_{\mathrm{G}}(95 \%$ CI $)$ & $\mathrm{r}_{\mathrm{C}}(95 \% \mathrm{CI})$ & $\mathrm{r}_{\mathrm{E}}(95 \%$ CI $)$ \\
\hline BMI-TC & $0.26(-1.00$ to 1.00$)$ & - & $0.06(-0.08$ to 0.20$)$ \\
BMI-HDL-C & $-0.13(-1.00$ to 1.00$)$ & - & $-0.06(-0.21$ to 0.08$)$ \\
BMI-LDL-C & $0.12(-1.00$ to 1.00$)$ & $0.46(-1.00$ to 1.00$)$ & $0.11(-0.04$ to 0.24$)$ \\
BMI-SBP & $0.67(0.19-1.00)^{*}$ & $0.11(-1.00$ to 1.00$)$ & $0.07(-0.08$ to 0.21$)$ \\
BMI-DBP & $0.56(0.14-1.00)^{*}$ & - & $0.15(0.005-0.29)^{*}$ \\
FPG-TC & - & $0.93(-1.00$ to 1.00$)$ & $0.17(0.06-0.28)^{*}$ \\
FPG-HDL-C & - & $0.01(-1.00$ to 1.00$)$ & $0.17(0.06-0.28)^{*}$ \\
FPG-LDL-C & - & - & $0.12(0.01-0.22)^{*}$ \\
FPG-SBP & $0.04(-1.00$ to 1.00$)$ & - & $0.20(0.07-0.30)^{*}$ \\
FPG-DBP & - & - & $0.12(0.01-0.24)^{*}$ \\
TC-HDL-C & $0.18(-0.20$ to 0.34$)$ & $0.93(-1.00$ to 1.00$)$ & $0.57(0.46-0.66)^{*}$ \\
TC-LDL-C & $0.83(0.35-0.91)^{*}$ & - & $0.92(0.90-0.94)^{*}$ \\
TC-SBP & $0.15(-0.15$ to 0.50$)$ & - & $0.04(-0.11$ to 0.18$)$ \\
TC-DBP & $0.25(0.02-0.52)^{*}$ & $-0.69(-0.99$ to 1.00$)$ & $0.07(-0.08$ to 0.21$)$ \\
HDL-C-LDL-C & $-0.06(-0.69$ to 0.23$)$ & - & $0.41(0.28 \text { to } 0.52)^{*}$ \\
HDL-C-SBP & $-0.09(-0.37$ to 0.09$)$ & $0.96(-1.00$ to 1.00$)$ & $0.17(0.02 \text { to } 0.31)^{*}$ \\
HDL-C-DBP & - & - & $0.12(-0.03$ to 0.27$)$ \\
LDL-C-SBP & $0.18(-0.21$ to 0.61$)$ & $0.22(-1.00$ to 1.00$)$ & $0.01(-0.14$ to 0.16$)$ \\
LDL-C-DBP & $0.24(-0.08$ to 0.58$)$ & - & $0.08(-0.07$ to 0.22$)$ \\
SBP-DBP & $0.92(0.86-1.00)^{*}$ & - & $0.63(0.54 \text { to } 0.71)^{*}$ \\
\hline *p $<0.05$. & & &
\end{tabular}

\section{Results}

The medium and $95 \%$ ranges for various phenotypes are shown in table 1. ICCs for MZ and DZ pairs are summarized in table 2. For all phenotypes, after adjusting for age and sex, $\mathrm{MZ}$ correlations were consistently larger than those in DZ twins suggesting genetic contributions. The correlation for TG failed to show significance between MZ and DZ twins. This may indicate no genetic influence (see model fitting) in TG.

\section{Univariate Genetic Analyses}

Table 3 shows the besting fitting models for all intermediate phenotypes. The AE model was selected as the best model for all phenotypes except for TG which has the CE model as the best. In all AE models, moderate to high heritability were estimated. Heritability ranged from 0.50 to 0.78 with the highest of 0.78 for weight ( $95 \%$ CI $0.75-0.78$ ), and the lowest of 0.50 for TC (95\% CI 0.38-0.60) (table 3).

\section{Bivariate Genetic Models}

Because BMI, WHR and weight are all obesity-related anthropometric traits, BMI was chosen as a representative to reduce the number of combinations. We also dropped TG in the bivariate modeling due to no genetic influence. This resulted in a total of 7 phenotypes (BMI, FPG, TC, HDL-C, LDL-C, SBP and DBP) to include in the bivariate analysis. Table 4 shows the genetic and environmental contributions to phenotypic correlations. The bivariate Cholesky decomposition revealed high genetic correlations between SBP and DBP $\left(\mathrm{r}_{\mathrm{G}}=0.92,95 \%\right.$ CI $\left.0.86-1\right)$ as well as TC and LDL-C $\left(\mathrm{r}_{\mathrm{G}}=\right.$
0.83, 95\% CI 0.35-0.91), modest genetic correlation between BMI and $\mathrm{SBP}\left(\mathrm{r}_{\mathrm{G}}=0.67,95 \% \mathrm{CI} 0.19-1\right)$ as well as BMI and DBP $\left(r_{G}=0.56,95 \%\right.$ CI $\left.0.14-1\right)$, and a weak but significant genetic correlation between TC and SBP $\left(\mathrm{r}_{\mathrm{G}}=0.25,95 \% \mathrm{CI}\right.$ 0.02-0.52). Significant contributions from unique environmental effects were estimated for the covariance between TC and HDL-C ( $\mathrm{r}_{\mathrm{E}}=0.57,95 \%$ CI $\left.0.46-0.66\right)$, TC and LDL-C $\left(r_{\mathrm{E}}=0.92,95 \%\right.$ CI 0.90-0.94), HDL-C and LDL-C $\left(r_{\mathrm{E}}=0.41\right.$, 95\% CI 0.28-0.52), and SBP and DBP ( $\mathrm{r}_{\mathrm{E}}=0.63,95 \% \mathrm{CI}$ $0.54-0.71)$. No common environmental correlations between phenotype pairs showed statistical significance.

\section{Discussion}

In the present study, we have assessed the heritability of 11 intermediate phenotypes covering glucose concentration, obesity, blood fat and blood pressure in Chinese twins. Ten phenotypes (WHR, BMI, weight, FPG, 2hPG, HDL-C LDL-C, TC, SBP and DBP) displayed significant moderate to high additive genetic determinations and unique environmental effects.

Obesity, and in particular abdominal obesity, is a known risk factor for the development of type 2 diabetes. The heritability estimates for absolute weight, WHR and BMI indicated a major genetic component in the development of obesity. The high heritability of BMI and absolute weight found in this study is in accordance with previous studies $[4,6-7,11$, 24-25]. Our heritability estimate for WHR is close to that of previous reports in Belgian [6] and Finnish twins [24], but higher than that reported in Danish twins $[11,25]$. The ob- 
served difference in heritability estimates could indicate a higher genetic dissection on WHR in the Chinese population as compared with Danes.

Our study estimated higher genetic effects in the total variation of FPG (71\%) and 2hPG (56\%) compared with those reported by other studies [3, 6-7, 9-11]. These estimates could reflect a stronger genetic component in glucose metabolism in the Chinese population. In contrast to other studies $[4,25]$ reporting a lower heritability of FPG when compared to $2 \mathrm{hPG}$, our heritability estimate for FPG is higher than that for $2 \mathrm{hPG}$. Whether the difference reflects an ethnic disparity requires further replication studies.

With regard to serum lipids, variations on TC, HDL-C and LDL-C were primarily explained by additive genetic factors, accounting for 50,58 and $60 \%$ of their total variances, with residual variances explained by unique environmental factors. In contrast to some previous studies [6-7, 9-11], we found no genetic effect for TG. However, our result is in agreement with two other twin studies conducted in China. Chen et al. [8] and Ren et al. [5] used twin correlation coefficients to estimate heritability of serum lipid for Chinese young and adult twins, and both reported no statistical significance for the TG phenotype in their Chinese samples. The consistent results on TG in Chinese studies could mean that there could be an ethnic difference in the genetics of TG phenotype.

By applying a multivariate model to the Danish twin data, Benyamin et al. [26] recently found that, in general, there are no common genetic and familial environmental factors behind the endophenotypes of metabolic syndrome. Our parameter estimates from the bivariate model (table 4) only showed significant genetic correlation between SBP and DBP, TC and LDL-C, and BMI and blood pressure, with the first two indicating pleiotropic genetic control over physiologically similar phenotypes. Of special interest is our discovery of significant genetic correlation between BMI and both systolic and diastolic blood pressures in Chinese which is consistent with the results obtained by Choh et al. [27] in American Samoan families. Significant phenotype correlation between BMI and blood pressure has been reported by multiple studies across populations [28]. The detection of significant genetic contribution to the phenotype correlation between BMI and blood pressure suggests the existence of pleiotropic genetic mechanisms behind the two phenotypes and across populations.

The current study is limited to univariate and bivariate analyses. As the sub-phenotypes are all related to obesity or metabolic syndrome, it would be interesting to apply multivariate analysis on selected variables to examine if functional clusters of phenotypes also share genetic mechanisms within functional/biological pathways. Furthermore, our samples in this study were collected in north China where composition of food and geographical environment are different from those in the southern part of the country. Given the importance of the environmental effect on metabolic phenotypes, similar twin-based studies conducted in south China should help to reveal the interplay between genetics and environment as a result of environmental adaptation for the same ethnic population.

\section{Acknowledgements}

This study was supported by the Novo Nordisk Foundation 2006 grant for Medical and Natural Science Research and partially by the National Natural Science Foundation of China (grant \#30872170).

\section{Disclosure Statement}

The authors declare no conflicts of interest.

\section{References}

1 Wang L, Kong L, Wu F, Bai Y, Burton R: Preventing chronic diseases in China. Lancet 2005;366: 1821-1824.

2 Cheng TO: Price of modernization of China. Circulation 2001;103:E103-132.

3 Schousboe K, Visscher PM, Henriksen JE, Hopper JL, Sørensen TIA, Kyvik KO: Twin study of genetic and environmental influences on glucose tolerance and indices of insulin sensitivity and secretion. Diabetologia 2003;46:1276-1283.

4 Schousboe K, Visscher PM, Erbas B, Kyvik KO, Hopper JL, Henriksen JE, Heitmann BL, Sørensen TIA: Twin study of genetic and environmental influences on adult body size, shape, and composition. Int J Obes 2004;28:39-48.

5 Ren T, Wu DF, Hu YH, Cao WH, Chan SY, Lu J, Zhai Y, Wu T, Li LM: The heritability analysis of the metabolic syndrome related characters in twins. Chin J Prev Contr Chron Noncommun Dis 2003; 11:13-15.
6 Souren NY, Paulussen AD, Loos RJ, Gielen M, Beunen G, Fagard R, Derom C, Vlietinck R, Zeegers MP: Anthropometry, carbohydrate and lipid metabolism in the East Flanders Prospective Twin Survey: heritabilities, Diabetologia 2007;50: 2107-2116.

7 Luo BF, Du L, Li JX, Pan BY, Xu JM, Chen J, Yin XY, Ren Y, Zhang F: Heritability of metabolic syndrome traits among healthy younger adult: a population-base study in China. J Med Genet 2009; 47:415-420.

8 Chen TJ, Ji CY, Pang ZC, Wang SJ, Hu YH, Qin Y: Heritability of serum lipids and lipoproteins and its related factors in the twins aged 5 to 19 years of china. Chin J Prev Med 2004:38:237-239.

9 Henneman P, Aulchenko YS, Frants RR, van Dijk KW, Oostra BA, van Duijn CM: Prevalence and heritability of the metabolic syndrome and its individual components in a Dutch isolate: the Erasmus Rucphen Family study. J Med Genet 2008;45:572577.
10 Lin HF, Boden-Albala B, Juo SH, Park.N: Heritabilities of the metabolic syndrome and its components in the Northern Manhattan Family Study. Diabetologia 2005;48:2006-2012.

11 Poulsen P, Vaag A, Kyvik K, Beck-Nielsen H: Genetic versus environmental aetiology of the metabolic syndrome among male and female twins. Diabetologia 2001;44:537-543.

12 Zhang S, Liu X, Yu Y, Hong X, Christoffel KK, Wang B, Tsai HJ, Li Z, Liu X, Tang G, Xing H, Brickman WJ, Zimmerman D, Xu X, Wang X: Genetic and environmental contributions to phenotypic components of metabolic syndrome: a population-based twin study. Obesity (Silver Spring) 2009;17:1581-1587.

13 Lee J, Chen L, Snieder H, Chen da F, Lee LM, Liu GF, Wu T, Tang X, Zhan SY, Cao WH, Lv J, Gao WJ, Hu YH: Heritability of obesity-related phenotypes and association with adiponectin gene polymorphisms in the Chinese national twin registry. Ann Hum Genet 2010;74:146-154. 
14 Abate N, Chandalia M: The impact of ethnicity on type 2 diabetes. J Diabetes Complications 2003;17:39-58.

15 Dagogo-Jack S: Ethnic disparities in type 2 diabetes: pathophysiology and implications for prevention and management. J Natl Med Assoc 2003;95 774-789.

16 Cockram CS, Woo J, Lau E, Chan JC, Chan AY, Lau J, Swaminathan R, Donna SP: The prevalence of diabetes mellitus and impaired glucose tolerance among Hong Kong Chinese adults of working age. Diabetes Res Clin Pract 1993;21:67-73.

17 Chan JCN: Heterogeneity of diabetes mellitus in the Hong Kong Chinese population. The Chinese University of Hong Kong-Prince of Wales Hospital Disbetes Research and Care Group. Hong Kong Med J 2000;6:77-84.

18 Thai AC, Yeo PPB, Lun KC, Highes K, Wang KW, Sothy SP, Lui KF, Ng WY, Cheah JS, Phoon WO, Lim P: Changing prevalence of diabetes mellitus in Singapore over a ten year period; in Vannasaeng S, Nitiyanant W, Chandraprasert S (eds): Epidemiology of Diabetes Mellitus. Proceedings of the International Symposium on Epidemiology of Diabetes Mellitus. Mankok, Crystal House Press, 1986, pp 63-67.
19 Chou P, Chen HH, Hsiao KJ: Community-based epidemiological study on diabetes in $\mathrm{Pu}-\mathrm{Li}$, Taiwan. Diabetes Care 1992;15:81-89.

20 Pang ZC, Ning F, Unger J, Johnson CA, Wang S, Guo Q,Cao WH, Lee LM: The Qingdao Twin Registry: a focus on chronic disease research. Twin Res Hum Genet 2006;9:758-762.

21 Tomsey CS, Kurtz M, Kist F, Hockensmith M, Call P: Comparison of PowerPlex 16, PowerPlex1.1/2.1, and ABI AmpfISTR Profiler Plus/COfiler for forensic use. Croat Med J 2001;42:239-243.

22 Neale MC: Mx: Statistical Modeling. Richmond, Department of Psychiatry, Medical College of Virginia, 1997.

23 Neale MC, Cardon LR: Methodology of Genetic Studies in Twins and Families. Dordrecht, Kluwer Academic Publishers, 1992.

24 Lehtovirta M, Kaprio J, Forsblom C, Eriksson J, Tuomilehto J, Groop L: Insulin sensitivity and insulin secretion in monozygotic and dizygotic twins. Diabetologia 2000;43:285-293.
25 Poulsen P, Kyvik KO, Vaag A, Beck-Nielsen H: Heritability of type II (non-insulin-dependent) diabetes mellitus and abnormal glucose tolerance - a population-based twin study. Diabetologia 1999; 42:139-145.

26 Benyamin B, Sørensen TIA, Schousboe K, Fenger M, Visscher PM, Kyvik KO: Are there common genetic and environmental factors behind the endophenotypes associated with the metabolic syndromes? Diabetologia 2007;50:1880-1888.

27 Choh AC, Gage TB, McGarvey ST, Comuzzie AG: Genetic and environmental correlations between various anthropometric and blood pressure traits among adult Samoans. Am J Phys Anthropol 2001;115:304-311.

28 Mufunda J: Body mass index and blood pressure: where are we now? J Hum Hypertens 2007;21:5-7. 